\title{
Index of Estimated Benefit from Lateral Lymph Node Dissection for Middle and Lower Rectal Cancer
}

\author{
MASAKATSU NUMATA, TOMOHIRO YAMAGUCHI, YUSUKE KINUGASA, AKIO SHIOMI, HIROYASU KAGAWA, \\ YUSHI YAMAKAWA, AKINOBU FURUTANI, SHOICHI MANABE and YUSUKE YAMAOKA \\ Division of Colon and Rectal Surgery, Shizuoka Cancer Center Hospital, Shizuoka, Japan
}

\begin{abstract}
Aim: To evaluate the clinical benefit from lateral lymph node dissection for middle and lower rectal cancer. Patients and Methods: A total of 229 patients who underwent bilateral lateral lymph node dissection during resection with curative intent for cT3-4 middle or lower rectal cancer from 2002 to 2013 were retrospectively reviewed. The index of estimated benefit from lymph node dissection for all, and each (common iliac, internal iliac and obturator), lateral lymph nodes were compared with the index for mesenteric lymph nodes (perirectal, intermediate and main lymph nodes). Results: The overall incidence of lateral lymph node metastasis was $14.0 \%$. The index for all lateral lymph nodes (10.6) was lower than perirectal lymph nodes (45.3), but higher than intermediate lymph nodes (4.8). Conclusion: Lateral lymph node dissection provides considerable clinical benefit, similar to the benefit provided by intermediate lymph node dissection.
\end{abstract}

The incidence of lateral lymph node (LN) metastasis from locally advanced middle and lower rectal cancer is reported to be $14.6-20.1 \%$. It is associated with increased local recurrence and poor overall survival (1-4). In Western countries, lateral $\mathrm{LN}$ metastasis is generally considered a metastatic disease and neoadjuvant chemoradiotherapy (CRT) combined with total mesorectal excision (TME) is widely used (5-7). On the contrary, the Japanese Classification of Colorectal Carcinoma (8) defines lateral LNs as regional LNs in the internal iliac (IILN), obturator (OLN) and common iliac (CILN) subregions. The Japanese Society for Cancer of the Colon and Rectum (JSCCR) Guidelines for the treatment of colorectal

Correspondence to: Tomohiro Yamaguchi, MD, PhD, Division of Colon and Rectal Surgery, Shizuoka Cancer Center Hospital, 1007 Shimonagakubo, Nagaizumi-cho, Sunto-gun, Shizuoka, 411-8777, Japan. Tel: +81 559895222, Fax: +81 559895783, e-mail: t.yamaguchi@scchr.jp

Key Words: Rectal cancer, lateral lymph node dissection, the index of estimated benefit from lymph node dissection. cancer (3) recommend mesorectal excision with lateral LN dissection (LLD) as standard treatment for locally advanced middle and lower rectal cancer. However, the prognostic benefit of LLD remains unclear.

In this study, the index of estimated benefit from LN dissection (IEBLD), unique method to assess the efficacy of LN dissection (9), was adopted to clarify the therapeutic value of LLD for locally advanced middle and lower rectal cancer.

\section{Patients and Methods}

Study design. A prospective colorectal database, which contains information on patients' characteristics, preoperative assessment, operative characteristics, postoperative complications, pathological characteristics and follow-up data, was used for analysis. From September 2002 to June 2013, a total of 389 patients who had been diagnosed with cT3-4 middle or lower rectal adenocarcinoma underwent mesorectal excision with LLD for curative intent at Shizuoka Cancer Center Hospital. First, 21 patients who received neoadjuvant CRT were excluded. Neoadjuvant CRT was used only when resection margin positivity was suspected based on preoperative computed tomography $(\mathrm{CT})$ and magnetic resonance imaging (MRI) findings. Subsequently, 24 patients who had prior treatment for any pelvic malignancy, 33 patients with unilateral LLD and 82 patients with sampling of lateral LNs were excluded. Finally, 229 patients who underwent bilateral LLD were included in this study (Figure 1).

Definition of mesenteric LNs. The Japanese Classification of Colorectal Carcinoma categorizes mesenteric LNs of the rectum into 3 subgroups: perirectal LNs (PLNs), intermediate LNs (ILNs) and main LNs (MLNs) (Figure 2) (8). The MLNs are defined as the LNs at the root of the inferior mesenteric artery (IMA). The ILNs are defined as the LNs around the IMA between the left colic artery and the terminal branch of the sigmoid artery. The JSCCR Guidelines for the treatment of colorectal cancer (3) recommend mesenteric LN dissection that includes the PLNs, ILNs and MLNs for cT2-4 or $\mathrm{cN}+$ rectal cancer.

Indications for LLD. LLD is indicated when the lower border of the tumor is located distal to the peritoneal reflection and has invaded beyond the muscularis propria (cT3-4) in accordance with the JSCCR Guidelines for the treatment of colorectal cancer (3). Patients without lateral $\mathrm{LN}$ metastasis on preoperative imaging who 
were aged over 76 years or at high-risk of postoperative complications due to comorbid conditions did not undergo LLD. In patients diagnosed with lateral $\mathrm{LN}$ metastasis on preoperative imaging who were aged over 76 years or at high-risk of preoperative complications due to comorbid conditions, unilateral (metastatic side) dissection or sampling of lateral LNs was performed. Patients aged 75 years or younger who were not at highrisk of postoperative complications underwent bilateral LLD.

Preoperative evaluation included digital rectal examination, colonoscopy, histological examination, CT, MRI and barium enema. Patients were staged using the tumor node metastasis (TNM) staging system in the seventh edition of the American Joint Committee on Cancer (AJCC) Cancer Staging Manual (10). Although the TNM staging system considers lateral LN metastasis as distant metastasis, the present study considers lateral LN metastasis as regional metastasis (4).

LLD operative procedure. Bilateral LLD, including bilateral complete dissection of CILNs, IILNs and OLNs, was performed after removal of the rectum (Figure 3). First, the ureter was identified and mobilized. To dissect the CILNs, the ventral and medial portions of the common iliac artery and vein were exteriorized from the aortic bifurcation. Dissection was performed along the blood vessel wall to the bifurcation of the internal and external iliac arteries. Next, IILNs, located between the internal iliac artery and the autonomic nerves (pelvic splanchnic nerves and pelvic plexus), were dissected. Finally, OLNs were dissected. The border between the internal iliac vessels and OLNs was separated. The lateral surface of the internal pudendal artery was exfoliated to Alcock's canal and the sacral nerves, as well as the coccygeal muscle were exposed. Obturator nerves were preserved and the obturator artery and vein were dissected. The lateral side of the obturator space was exposed to the pelvic wall along the obturator fascia, while the inside of the pelvic wall was dissected to the lateral side of the internal iliac artery. The proximal side was dissected en bloc to the bifurcation of the internal and external iliac arteries (11).

Up to December 2011, when robotic-assisted laparoscopic surgery began at our Institution, rectal cancer surgery with LLD was performed using the open method. Since January 2012, rectal cancer surgery with LLD has also been performed using robotic-assisted LLD $(11,12)$. Laparoscopic LLD was adopted for patients who prefer not to have robotic or open surgery. The lateral $\mathrm{LN}$ resection area was the same regardless of surgical approach.

Evaluation of the therapeutic value of $L L D$. We adopted the IEBLD to assess the efficacy of $\mathrm{LN}$ dissection for each $\mathrm{LN}$ region. This index was proposed by Sasako et al. (9) in 1995. It is calculated by multiplying the frequency of LN metastasis in each region by the 5 -year survival rate of patients with positive LNs in each region. $\mathrm{LN}$ metastasis was recorded separately for each $\mathrm{LN}$ region. In this study, the IEBLD was calculated for all lateral LNs, CILNs, IILNs and OLNs. The IEBLD for all lateral LNs represents the benefit from bilateral complete dissection of CILNs, IILNs and OLNs, which was calculated by multiplying the frequency of any lateral LN metastasis by the 5-year survival rate of patients with any lateral LN metastasis. Then, the IEBLD for mesenteric LNs, including all mesenteric LNs, PLNs, ILNs and MLNs, was also calculated and compared to the IEBLD for lateral LNs. Because it is impossible to determine a cut-off level as a definite indication for $\mathrm{LN}$ dissection

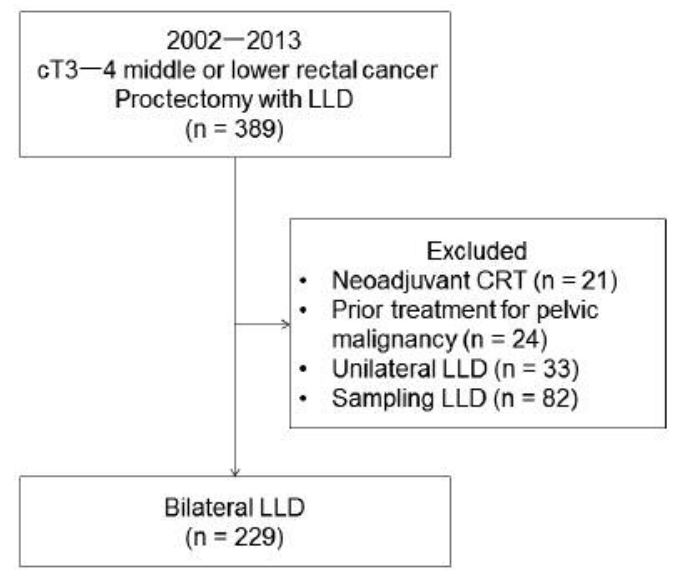

Figure 1. Consort diagram. LLD, Lateral lymph node dissection, CRT, chemoradiotherapy.

(13), the IEBLD of mesenteric LNs, which are routinely dissected in locally advanced rectal cancer, was used as reference. Previous articles dealing with IEBLD also used the IEBLD of routinely dissected LNs as a reference (14-18).

Patients' follow-up. Patients who were diagnosed with pStage III disease underwent adjuvant chemotherapy after the operation for 6 months. Tumor markers were measured every 3 months for 3 years and then every 6 months until 5 years. CT scans were performed every 6 months for 5 years. All study protocols were approved by our Institutional Review Board (No. 28-J58-28-1-3) and informed consent was obtained from all patients.

Statistical analysis. All statistical analyses were performed using EZR (Saimata Medical Center, Jichi Medical University, Saitama, Japan) (19). All $p$-values were 2-sided and $p$-values less than 0.05 were considered statistically significant.

\section{Results}

Patients' demographic characteristics and pathological findings. Table I summarizes the demographic characteristics and pathological findings of the 229 study patients. The open approach was used in 199 patients (86.9\%), compared to robotic in 28 patients $(12.2 \%)$ and laparoscopic in 2 patients $(0.9 \%)$. Regarding pT stage, 188 patients $(82.1 \%)$ had pT3 or pT4 disease. As for pN stage, 122 patients $(53.3 \%)$ had $\mathrm{pN}+$ disease. Lateral LN metastasis was observed in 32 patients $(14.0 \%)$. As for pathological stage, 194 patients $(84.8 \%)$ were diagnosed with Stage II or III disease, while 30 patients $(13.1 \%)$ had Stage I disease and 5 patients $(2.1 \%)$ had Stage IV disease. The median number of harvested LNs was 48 . The median number of harvested total lateral LNs, CILNs, IILNs and OLNs was $21,3,5$ and 13 , respectively. R0 resection was achieved in all patients. 


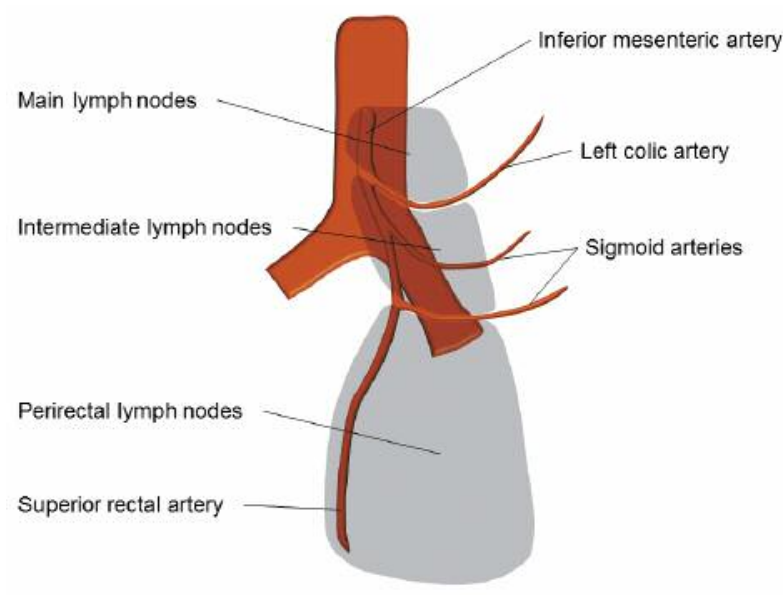

Figure 2. Schematic image showing the location of the mesenteric lymph nodes, including perirectal lymph nodes, intermediate lymph node (ILNs) and main lymph nodes (MLNs) around the inferior mesenteric artery.

Frequency of $L N$ metastasis and IEBLD for each $L N$. Table II presents the frequency of metastasis, 5-year overall survival rate of patients with nodal involvement and IEBLD for each type of mesenteric and lateral LNs. The overall incidence of metastasis to mesenteric and lateral $\mathrm{LNs}$ was $50.7 \%$ and $14.0 \%$, respectively. Among 113 patients without metastasis to mesenteric LNs, the metastasis to lateral LNs was observed in 6 patients $(5.3 \%)$. The incidence of metastasis to lateral LNs $(14.0 \%)$ was higher than that of ILNs $(5.7 \%)$. The 5-year overall survival rate for patients with lateral $\mathrm{LN}$ metastasis was significantly worse than the rate for patients without lateral LN metastasis (75.7\% vs. 95.2\%, $p=0.0002)$ (Figure 4). However, the 5-year overall survival rate was not significantly different between patients with ILN metastasis and lateral LN metastasis $(84.6 \%$ vs. $75.7 \%, p=0.88)$ (Figure 5). The IEBLD for all lateral LNs (10.6) was higher than that for ILNs (4.8). Among the lateral subregions, OLNs and IILNs had nearly identical IEBLDs. CILNs had the same IEBLD (0.5) as MLNs (0.5). Median follow-up for all patients was 60.8 months.

\section{Discussion}

Middle and lower rectal cancer are considered to have 2 lymphatic pathways: superior and lateral. Regarding the superior pathway, LN metastasis arises from the perirectal area and spreads along the superior rectal artery to the IMA $(20,21)$. In Japan, during $\mathrm{LN}$ dissection for the superior pathway, ligation at the root of the IMA is usually performed for patients with locally advanced rectal cancer (3). Previous literature from a Western country also described central vascular ligation (CVL) with the mesenteric dissection up to MLNs as an important technique for improving oncologic

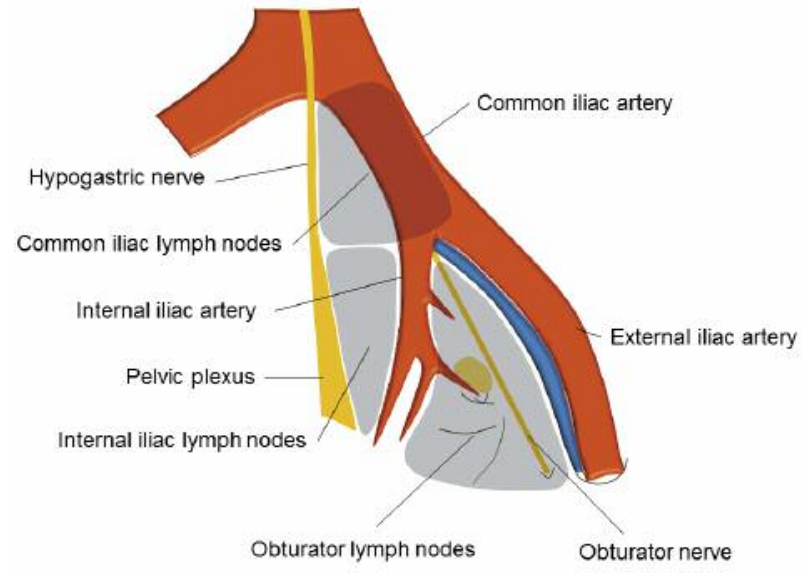

Figure 3. Schematic image showing the location of the left lateral lymph nodes, including the common iliac lymph nodes (CILNs), internal iliac lymph nodes (IILNS) and obturator lymph nodes (OLNS).

outcomes (22). On the other hand, the strategy for lateral $\mathrm{LN}$ metastasis is different in Japan and Western countries. In Western countries, TME with neoadjuvant CRT has become the standard treatment for locally advanced rectal cancer. On the other hand, mesorectal excision with LLD is the standard treatment for locally advanced middle and lower rectal cancer in Japan. However, the efficacy of LLD has not been fully evaluated in a prospective study. The recommendation for LLD in the JSCCR Guidelines for the treatment of colorectal cancer is based on a nation-wide observational study. Sugihara et al. found that the incidence of lateral LN metastasis was $20.1 \%$ among patients who underwent LLD. They found that the risk of local recurrence decreased by $50.3 \%$ and the 5-year survival rate improved by $8 \%$ when LLD was performed for T3-4 middle and lower rectal cancer (2). In addition, Akiyoshi et al. reported that patients with lateral LN metastasis localized to IILNs and N2a patients have a similar prognosis, while patients with metastasis to lateral LNs other than IILNs have a poorer prognosis. However, their prognosis is similar to that of $\mathrm{N} 2 \mathrm{~b}$ patients and better than that of Stage IV patients who underwent curative resection (4). These 2 studies are retrospective in nature and have the limitations inherent to this type of study design. Recently, a multi-center randomized controlled trial comparing mesorectal excision with and without LLD for clinical Stage II or III middle and lower rectal cancer was carried out in Japan. In the analysis of short-term outcomes, Fujita et al. and Saito et al. reported that the morbidity and urogenital dysfunction were not significantly different between mesorectal excision alone and mesorectal excision with LLD, which demonstrated the safety and feasibility of $\operatorname{LLD}(23,24)$. The results of the primary analysis have not 
Table I. Demographic characteristics and pathological findings of our patient cohort $(n=229)$.

\begin{tabular}{|c|c|}
\hline Parameters & Number \\
\hline Age (years) [median (range)] & $61(26-79)$ \\
\hline \multicolumn{2}{|l|}{ Gender } \\
\hline Male & $164(71.6 \%)$ \\
\hline Female & $65(28.4 \%)$ \\
\hline \multicolumn{2}{|l|}{ ASA classification } \\
\hline Class 1 & $63(27.5 \%)$ \\
\hline Class 2 & $163(71.2 \%)$ \\
\hline Class 3 & $3(1.3 \%)$ \\
\hline \multicolumn{2}{|l|}{ Type of operation } \\
\hline Low anterior resection & $119(52.0 \%)$ \\
\hline Intersphincteric resection & $38(16.5 \%)$ \\
\hline Abdominoperineal resection & $56(24.5 \%)$ \\
\hline Total pelvic exenteration & $16(7.0 \%)$ \\
\hline \multicolumn{2}{|l|}{ Approach } \\
\hline Open & $199(86.9 \%)$ \\
\hline Robotic & $28(12.2 \%)$ \\
\hline Laparoscopic & $2(0.9 \%)$ \\
\hline Tumor size $(\mathrm{cm})$ [median (range)] & $5.0(2.0-13.0)$ \\
\hline \multicolumn{2}{|l|}{ Histology } \\
\hline $\begin{array}{l}\text { Well- or moderately differentiated } \\
\text { tubular adenocarcinoma }\end{array}$ & $216(94.3 \%)$ \\
\hline $\begin{array}{l}\text { Poorly differentiated or mucinous } \\
\text { adenocarcinoma }\end{array}$ & $13(5.7 \%)$ \\
\hline \multicolumn{2}{|l|}{ pT } \\
\hline $\mathrm{T} 2$ & $41(17.9 \%)$ \\
\hline $\mathrm{T} 3$ & $160(69.9 \%)$ \\
\hline $\mathrm{T} 4$ & $28(12.2 \%)$ \\
\hline \multicolumn{2}{|l|}{$\mathrm{pN}$} \\
\hline No & $107(46.7 \%)$ \\
\hline $\mathrm{N} 1$ & $65(28.4 \%)$ \\
\hline $\mathrm{N} 2$ & $57(24.9 \%)$ \\
\hline \multicolumn{2}{|c|}{ Pathological lateral lymph node metastasis } \\
\hline Negative & $197(86.0 \%)$ \\
\hline Positive & $32(14.0 \%)$ \\
\hline \multicolumn{2}{|l|}{ pStage } \\
\hline I & $30(13.1 \%)$ \\
\hline II & $75(32.8 \%)$ \\
\hline III & $119(52.0 \%)$ \\
\hline IV & $5(2.1 \%)$ \\
\hline \multicolumn{2}{|c|}{ Number of lymph nodes harvested [median (range)] } \\
\hline All lymph nodes & $48(11-155)$ \\
\hline Mesenteric lymph nodes & $31(10-144)$ \\
\hline PLNs & $22(5-138)$ \\
\hline ILNs & $8(0-18)$ \\
\hline MLNs around the IMA & $3(0-12)$ \\
\hline Lateral lymph nodes & $21(7-62)$ \\
\hline CILNs & $3(1-11)$ \\
\hline IILNs & $5(2-31)$ \\
\hline OLNs & $13(5-60)$ \\
\hline Positive resection margin & $0(0.0 \%)$ \\
\hline Adjuvant chemotherapy & $93(40.6 \%)$ \\
\hline
\end{tabular}

ASA, American Society of Anesthesiologists; PLN, perirectal lymph node; ILN, intermediate lymph node; MLN, main lymph node; IMA, inferior mesenteric artery; CILN, common iliac lymph node; IILN, internal iliac lymph node; OLN, obturator lymph node. yet been published. However, the efficacy of LLD for all cT3-4 cancers will not be clarified by this trial because patients with clinical lateral LN metastasis detected with preoperative imaging were excluded. Thus, the present study attempted to evaluate the therapeutic value of LLD relative to the value of dissecting mesenteric LNs in patients with cT3-4 middle and lower rectal cancer, including those with clinical lateral LN metastasis.

The Japanese Classification of Colorectal Carcinoma (8) distinguishes ILNs from PLNs, partly because metastasis to ILNs is associated with poorer prognosis than metastasis to PLNs (25). Japanese surgeons generally consider ILN dissection to be essential because the JSCCR Guidelines for the treatment of colorectal cancer recommend $\mathrm{LN}$ dissection up to ILNs even for T1 rectal cancer. Recently, accompanied by the widespread acceptance of the CVL technique (22), the importance of ILN dissection has become globally accepted.

The present study showed that the overall incidence of lateral LN metastasis was $14.0 \%$, which was in accordance with previous reports (14.6-20.1\%) (1-4). The IEBLD for all lateral LNs reached 10.6, which was higher than the IEBLD for ILNs $(4.8 \%)$. This result was due to the fact that (i) survival was achieved after LLD in patients with lateral LN metastasis, and (ii) the rate of lateral LN metastasis was higher than that of ILN metastasis. This result is consistent with a previous report by Ueno et al. (13). They reviewed 244 patients with middle or lower rectal cancer who underwent LLD using IEBLD with the objective of identifying parameters related to prognostic benefit. They found that the IEBLD for lateral LNs (9.9\%) was higher than the IEBLD for LNs around the superior rectal artery (4.2\%).

Information about the distribution of lateral $\mathrm{LN}$ metastasis is another important result of the present study. The evaluation of metastasis to each subregion using data from a single center has the advantage of avoiding region-to-region migration because the borders around each lateral LN group may differ by institution, which is affected by surgical technique or anatomical understanding. The present study revealed that the rates of IILN (7.4\%) and OLN (8.3\%) metastasis were similar. Among 32 patients with lateral LN metastasis, 31 (96.9\%) patients had metastasis to either IILNs or OLNs. There have been very few studies demonstrating the distribution of lateral LN metastasis. Ueno et al. (13), who divided the IILNs into proximal and distal IILNs based on the superior vesicular artery as a landmark, reported that the incidence of metastasis to any lateral LNs, proximal IILNs, distal IILNs and OLNs was $16.8 \%, 6.1 \%, 9.4 \%$ and $4.1 \%$, respectively. They also described the IILNs and OLNs as a 'vulnerable field' because $87.8 \%$ of patients with lateral LN metastasis had metastasis in this field. Yokoyama et al. analyzed 131 consecutive patients with locally advanced middle or lower rectal cancer who underwent LLD. The incidence of metastasis to any lateral LNs, IILNs and OLNs 


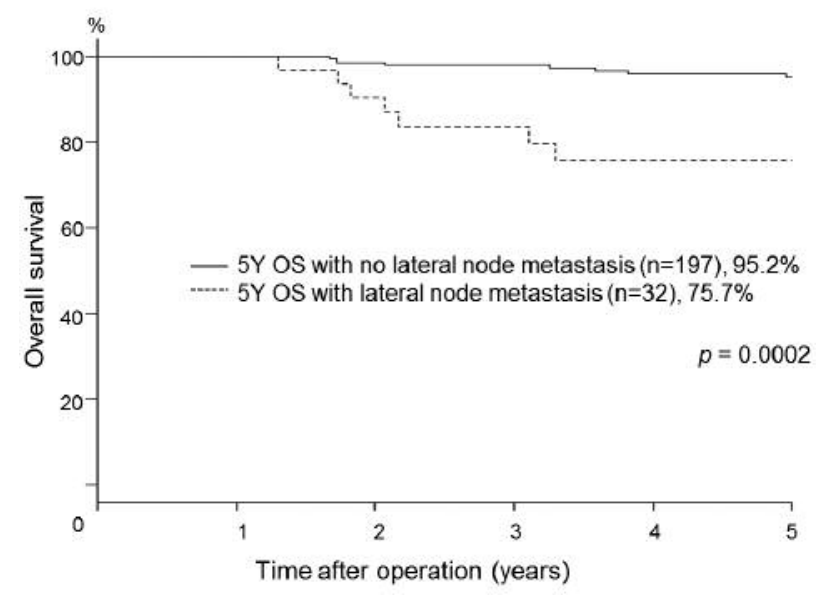

Figure 4. Five-year overall survival in patients with and without lateral lymph node metastasis. 5 Y OS, Five-year overall survival.

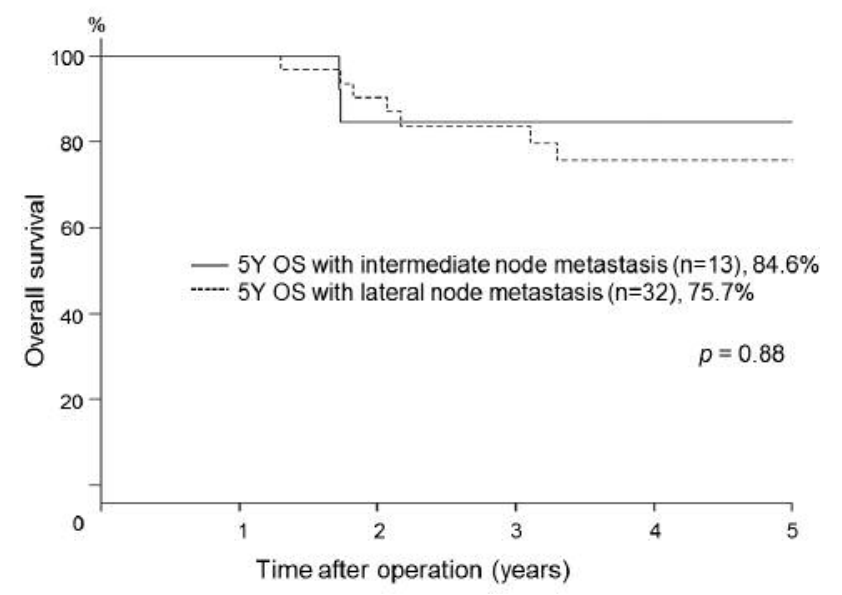

Figure 5. Five-year overall survival in patients with intermediate or lateral lymph node metastasis. 5 Y OS, Five-year overall survival.

Table II. Frequency of metastasis, 5-year survival and IEBLD for each type of lymph node $(n=229)$.

\begin{tabular}{lccc}
\hline Type of lymph node & $\begin{array}{c}\text { Number of patients } \\
\text { with metastasis }\end{array}$ & $\begin{array}{c}\text { 5-year overall survival rate among } \\
\text { patients with metastasis }\end{array}$ & IEBLD \\
\hline Mesenteric lymph nodes & $116(50.7 \%)$ & $89.3 \%$ & 45.3 \\
PLNs & $116(50.7 \%)$ & $89.3 \%$ & 45.3 \\
ILNs & $13(5.7 \%)$ & $84.6 \%$ & 5.8 \\
MLNs around the IMA & $2(0.9 \%)$ & $70.0 \%$ & 0.5 \\
Lateral lymph nodes & $32(14.0 \%)$ & $50.0 \%$ & 0.6 \\
CILNs & $2(0.9 \%)$ & $73.5 \%$ & 5.4 \\
IILNs & $17(7.4 \%)$ & $75.8 \%$ & 6.3 \\
OLNs & $19(8.3 \%)$ & & \\
\hline
\end{tabular}

IEBLD, Index of estimated benefit from lymph node dissection; PLN, perirectal lymph node; ILN, intermediate lymph node; MLN, main lymph node; IMA, inferior mesenteric artery; CILN, common iliac lymph node; IILN, internal iliac lymph node; OLN, obturator lymph node.

was $19.1 \%, 12.2 \%$ and $9.9 \%$, respectively. They also found that $96.1 \%$ of patients with lateral LN metastasis had metastasis to either IILNs or OLNs (26). The present work and the aforementioned previous studies found that most lateral LN metastases occur in the IILNs or OLNs. Both prior studies have demonstrated a higher incidence of IILN metastasis than OLN metastasis, which is not consistent with our results. This may be explained by region-to-region migration, as previously mentioned.

Another noteworthy finding of the present study was that the IEBLD for OLNs was not inferior to the IEBLD for IILNs. In the present work, the 5-year overall survival rate and IEBLD of patients with metastasis to OLNs were comparable to those with IILN metastasis. This result supports the fact that Japanese surgeons consider OLN metastasis to be regional LN metastasis, whereas the seventh edition of the AJCC Cancer Staging Manual considers OLN metastasis to be distant disease. The IEBLD for CILNs and MLNs were identical $(0.5 \%)$. However, there were only a few patients with metastasis to these subregions $(0.9 \%$ and $0.9 \%$ ), which made it difficult to specify the therapeutic value of dissecting these subregions.

There are several potential limitations in the present study. First, there might be a selection bias in which patients received LLD. Patients without lateral LN metastasis on preoperative imaging over 76 years of age or at high-risk of postoperative complications due to comorbid conditions did not undergo LLD. This selection bias may affect the incidence of lateral LN metastasis and overall survival. The second limitation is that the present data excluded 21 rectal cancer patients with neoadjuvant CRT as this approach has been reported to significantly decrease the number of harvested LNs (27), which 
might affect the number of harvested LNs and incidence of metastasis. The third limitation is sample size. The patient cohort of the present study included 229 patients with 32 patients having metastasis to lateral LNs. This number may not be enough to present the 5-year overall survival rate of patients with metastasis to lateral LNs. With these limitations, this retrospective study has to be considered as complementary research for revealing the clinical benefit of LLD.

In conclusion, the present study suggests that patients with locally advanced middle and lower rectal cancer can receive similar therapeutic benefit from LLD and ILN dissection. A prospective study is needed to confirm the efficacy of LLD for middle and lower rectal cancer.

\section{References}

1 Moriya Y, Hojo K, Sawada T and Koyama Y: Significance of lateral node dissection for advanced rectal carcinoma at or below the peritoneal reflection. Dis Colon Rectum 32(4): 307-315, 1989.

2 Sugihara K, Kobayashi H, Kato T, Mori T, Mochizuki H, Kameoka S, Shirouzu K and Muto T: Indication and benefit of pelvic sidewall dissection for rectal cancer. Dis Colon Rectum 49(11): 1663-1672, 2006.

3 Watanabe T, Itabashi M, Shimada Y, Tanaka S, Ito Y, Ajioka Y, Hamaguchi T, Hyodo I, Igarashi M, Ishida H, Ishiguro M, Kanemitsu Y, Kokudo N, Muro K, Ochiai A, Oguchi M, Ohkura Y, Saito Y, Sakai Y, Ueno H, Yoshino T, Fujimori T, Koinuma N, Morita T, Nishimura G, Sakata Y, Takahashi K, Takiuchi H, Tsuruta O, Yamaguchi T, Yoshida M, Yamaguchi N, Kotake K and Sugihara K: Japanese society for cancer of the colon and rectum (jsccr) guidelines 2010 for the treatment of colorectal cancer. Int J Clin Oncol 17(1): 1-29, 2012.

4 Akiyoshi T, Watanabe T, Miyata S, Kotake K, Muto T and Sugihara K: Results of a Japanese nationwide multi-institutional study on lateral pelvic lymph node metastasis in low rectal cancer: Is it regional or distant disease? Ann Surg 255(6): 11291134, 2012.

5 Kusters M, Beets GL, van de Velde CJ, Beets-Tan RG, Marijnen CA, Rutten HJ, Putter H and Moriya Y: A comparison between the treatment of low rectal cancer in Japan and the Netherlands, focusing on the patterns of local recurrence. Ann Surg 249(2): 229-235, 2009.

6 Georgiou P, Tan E, Gouvas N, Antoniou A, Brown G, Nicholls RJ and Tekkis P: Extended lymphadenectomy versus conventional surgery for rectal cancer: A meta-analysis. Lancet Oncol 10(11): 1053-1062, 2009.

7 Yano $\mathrm{H}$ and Moran BJ: The incidence of lateral pelvic side-wall nodal involvement in low rectal cancer may be similar in Japan and the West. Br J Surg 95(1): 33-49, 2008.

8 Japanese Society for Cancer of the Colon and Rectum. Japanese Classification of Colorectal Carcinoma, 2nd English edn. Tokyo, Japan, Kanehara Corp., 2009.

9 Sasako M, McCulloch P, Kinoshita T and Maruyama K: New method to evaluate the therapeutic value of lymph node dissection for gastric cancer. Br J Surg 82(3): 346-351, 1995.

10 Edge S, Byrd DR, Compton CC, Fritz AG, Greene FL and Trotti A: AJCC cancer staging manual. 7 edn. Supringer: New York, 2010.
11 Kagawa H, Kinugasa Y, Shiomi A, Yamaguchi T, Tsukamoto S, Tomioka H, Yamakawa Y and Sato S: Robotic-assisted lateral lymph node dissection for lower rectal cancer: Short-term outcomes in 50 consecutive patients. Surg Endosc 29(4): 995$1000,2015$.

12 Yamaguchi T, Kinugasa Y, Shiomi A, Tomioka H and Kagawa $\mathrm{H}$ : Robotic-assisted laparoscopic versus open lateral lymph node dissection for advanced lower rectal cancer. Surg Endosc 30(2): 721-728, 2015.

13 Ueno H, Mochizuki H, Hashiguchi Y, Ishiguro M, Miyoshi M, Kajiwara Y, Sato T, Shimazaki H and Hase K: Potential prognostic benefit of lateral pelvic node dissection for rectal cancer located below the peritoneal reflection. Ann Surg 245(1): 80-87, 2007.

14 Watanabe M, Kinoshita T, Enomoto N, Shibasaki H and Nishida $\mathrm{T}$ : Clinical significance of splenic hilar dissection with splenectomy in advanced proximal gastric cancer: An analysis at a single institution in Japan. World J Surg 40(5): 1165-1171, 2016.

15 Goto H, Tokunaga M, Miki Y, Makuuchi R, Sugisawa N, Tanizawa Y, Bando E, Kawamura T, Niihara M, Tsubosa Y and Terashima M: The optimal extent of lymph node dissection for adenocarcinoma of the esophagogastric junction differs between Siewert type II and Siewert type III patients. Gastric Cancer 18: 375-381, 2015.

16 Peng J, Wang WP, Yuan Y, Hu Y, Wang Y and Chen LQ: Optimal extent of lymph node dissection for Siewert type II esophagogastric junction adenocarcinoma. Ann Thorac Surg 100(1): 263-269, 2015.

17 Kosugi S, Kawaguchi Y, Kanda T, Ishikawa T, Sakamoto K, Akaike H, Fujii $\mathrm{H}$ and Wakai T: Cervical lymph node dissection for clinically submucosal carcinoma of the thoracic esophagus. Ann Surg Oncol 20(12): 4016-4021, 2013.

18 Kuroda H, Sakao Y, Mun M, Uehara H, Nakao M, Matsuura Y, Mizuno T, Sakakura N, Motoi N, Ishikawa Y, Yatabe Y, Nakagawa $\mathrm{K}$ and Okumura $\mathrm{S}$ : Lymph node metastases and prognosis in left upper division non-small cell lung cancers: The impact of interlobar lymph node metastasis. PLoS One 10(8): e0134674, 2015.

19 Kanda Y: Investigation of the freely available easy-to-use software 'EZR' for medical statistics. Bone Marrow Transplant 48(3): 452-458, 2013.

20 Grinnell RS: The lymphatic and venous spread of carcinoma of the rectum. Ann Surg 116(2): 200-216, 1942.

21 Heald RJ, Husband EM and Ryall RD: The mesorectum in rectal cancer surgery-the clue to pelvic recurrence? Br J Surg 69(10): 613-616, 1982.

22 West NP, Kobayashi H, Takahashi K, Perrakis A, Weber K, Hohenberger W, Sugihara K and Quirke P: Understanding optimal colonic cancer surgery: Comparison of Japanese D3 resection and European complete mesocolic excision with central vascular ligation. J Clin Oncol 30(15): 1763-1769, 2012.

23 Fujita S, Akasu T, Mizusawa J, Saito N, Kinugasa Y, Kanemitsu Y, Ohue M, Fujii S, Shiozawa M, Yamaguchi T and Moriya Y: Postoperative morbidity and mortality after mesorectal excision with and without lateral lymph node dissection for clinical stage II or stage III lower rectal cancer (JCOG0212): Results from a multicentre, randomised controlled, non-inferiority trial. Lancet Oncol 13(6): 616-621, 2012. 
24 Saito S, Fujita S, Mizusawa J, Kanemitsu Y, Saito N, Kinugasa Y, Akazai Y, Ota M, Ohue M, Komori K, Shiozawa M, Yamaguchi T, Akasu T and Moriya Y: Male sexual dysfunction after rectal cancer surgery: Results of a randomized trial comparing mesorectal excision with and without lateral lymph node dissection for patients with lower rectal cancer: Japan clinical oncology group study JCOG0212. Eur J Surg Oncol 42(12): 1851-1858, 2016.

25 Kobayashi $H$, Ueno $H$, Hashiguchi $Y$ and Mochizuki $H$ : Distribution of lymph node metastasis is a prognostic index in patients with stage III colon cancer. Surgery 139(4): 516-522, 2006.

26 Yokoyama S, Takifuji K, Hotta T, Matsuda K, Watanabe T, Mitani Y, Ieda J and Yamaue H: Survival benefit of lateral lymph node dissection according to the region of involvement and the number of lateral lymph nodes involved. Surg Today 44(6): 1097-1103, 2014.

27 Wichmann MW, Muller C, Meyer G, Strauss T, Hornung HM, Lau-Werner U, Angele MK and Schildberg FW: Effect of preoperative radiochemotherapy on lymph node retrieval after resection of rectal cancer. Arch Surg 137(2): 206-210, 2002.

Received February 1, 2017

Revised April 5, 2017

Accepted April 6, 2017 